\title{
Retroperitoneal cystic lymphangioma
}

\author{
Ali Aminian · Ali Jafarian · Rasoul Mirsharifi · Sima Moulavi · Habibollah Dashti · Fouzeyah A. H. Ali
}

Received: 18 April 2008 / Accepted: 18 May 2008

A 50-year-old man presented with vague abdominal discomfort and progressive abdominal swelling since 8 months. There was no history of previous medical and surgical problems including trauma. The only positive finding on physical examination was the presence of a large fixed left sided abdominal mass. Routine laboratory tests, amylase, tumor markers and hydatid serology were in normal limits. CT scan showed a large cystic abdominal lesion (Figs. 1, 2).

Laparotomy revealed a large retroperitoneal cystic lesion in close relations to pancreas, left kidney, ureter, duodenum, colon and aorta. The thin wall of the lesion rendered it liable to rupture and required handling with extreme care. The lesion was excised intact. Recovery was uneventful. Histopathology showed abundant lymphocytes with lymphoid follicle formation and dilated thin wall lymphatic channels, compatible with retroperitoneal cystic lymphangioma.

\section{Discussion}

Cystic lesions of the retroperitoneum can be categorized as either neoplastic or nonneoplastic. Neoplastic lesions

\footnotetext{
A. Aminian ${ }^{1} \cdot$ A. Jafarian ${ }^{1} \cdot$ R. Mirsharifi ${ }^{1}$.

S. Moulavi ${ }^{1}$ - H. Dashti ${ }^{1}$ F. A. H. Ali ${ }^{2}$

${ }^{1}$ Department of General Surgery,

Tehran University of Medical Sciences,

Tehran, Iran

${ }^{2}$ Department of Radiology,

Mubarak Al-Kabeer Hospital,

Kuwait
}

\section{A. Aminian $(\bowtie)$}

$\mathrm{Ph}:+98 / 9122054635$

Fax: $+98 / 21 / 66937185$

e-mail: aliaminian@doctor.com include cystic lymphangioma, cystic teratoma, cystic mesothelioma, epidermoid cyst, bronchogenic cyst, pseudomyxoma retroperitonei, necrotic neoplasms and undifferentiated sarcoma. Non-neoplastic lesions include pancreatic pseudocyst, nonpancreatic pseudocyst, hydatid cyst, lymphocele, urinoma, and hematoma [1].

Lymphangiomas are extremely rare benign neoplasms of the lymphatic system. They can occur in any location

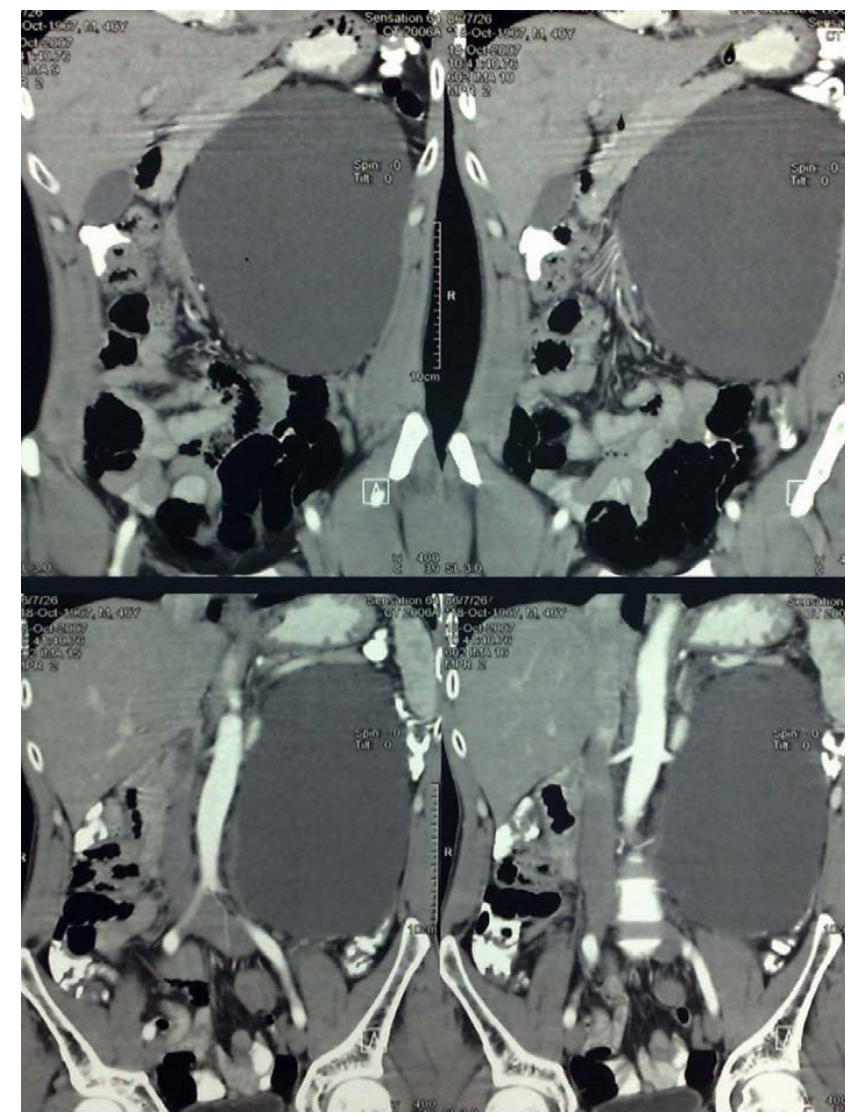

Fig. 1 CT scan: a large cystic abdominal lesion 


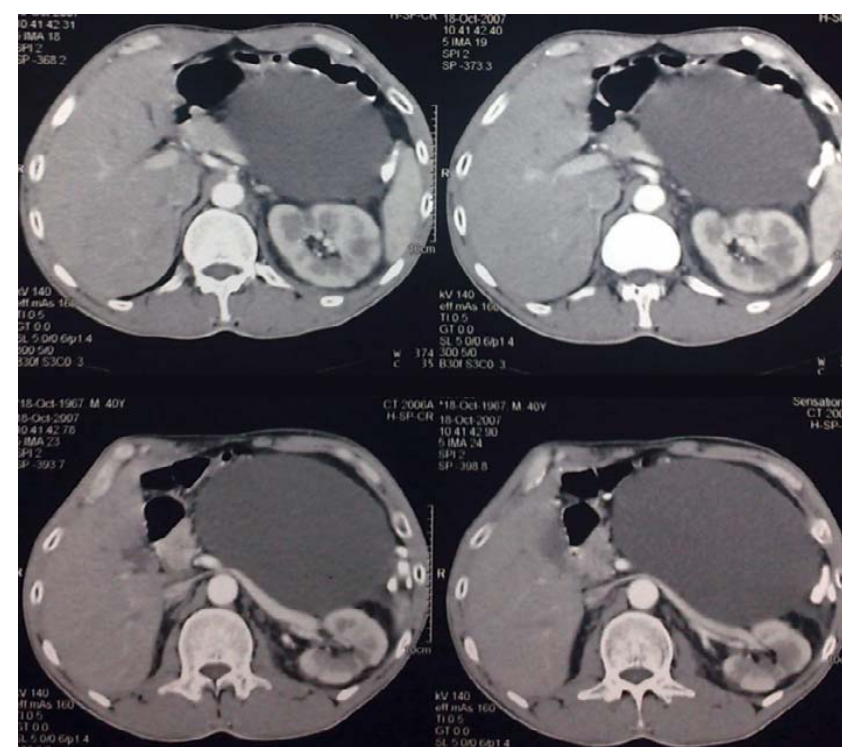

Fig. 2 CT scan: a large cystic abdominal lesion

where lymphatics are normally found. Head and neck lymphangiomas (cystic hygromas) account for $75 \%$ of reported cases. Retroperitoneal lymphangiomas account for approximately $1 \%$ of all lymphangiomas. One hundred and eighty-five cases were reported in a review of international literature $[2,3]$.

Intra-abdominal lymphangiomas may arise from the mesentery, omentum or retroperitoneum and tend to grow slowly within the abdomen. The majority is asymptomatic and discovered incidentally on abdominal imaging for other conditions. Symptoms are usually related to its size.
Approximately $40 \%$ of cases present with symptoms of obstruction. Other symptoms include ascites and intermittent fever. Rarely, bleeding or rupture will cause an acute abdomen $[2,3]$.

Preoperative diagnosis of lymphangiomas is rare prior to exploration. At CT scan, cystic lymphangioma typically appears as a large, thin-walled, multiseptate cystic mass. An elongated shape and a crossing from one retroperitoneal compartment to an adjacent one are characteristic of the mass [1].

Outcomes following complete resection of retroperitoneal lymphangiomas are generally good. Surgery is often required for symptom control or diagnosis. Recurrence of symptoms with incomplete excision is possible. Dissemination in the retroperitoneum is very rare but potentially a fatal complication. Injection of sclerosing agents into lymphangiomas has been described in nonsurgical candidates. However, induration of the cyst and infection often complicate these procedures [3].

\section{References}

1. Yang DM, Jung DH, Kim H, Kang JH, Kim SH, Kim JH, Hwang HY (2004) Retroperitoneal cystic masses: CT, clinical, and pathologic findings and literature review. Radiographics 24:1353-1365

2. Hauser H, Mischinger HJ, Beham A, Berger A, Cerwenka H, Razmara J, Fruhwirth H, Werkgartner G (1997) Cystic retroperitoneal lymphangiomas in adults. Eur J Surg Oncol 23:322-326

3. Cherk M, Nikfarjam M, Christophi C (2006) Retroperitoneal lymphangioma. Asian J Surg 29:51-54 